\title{
ALISHER NAVOIYNING “TARIXI MULUKI AJAM" ASARIDA ZOLIM SHOHLAR TASVIRI
}

Iroda PARDAYEVA*

\begin{abstract}
Annotatsiya
«Tarixi Muluki Ajam» Navoiyning tarixiy asari bo'lish bilan birga unda unda buyuk mutafakkirning adolatli shoh va davlat, zolim shohlar to'g'risidagi qarashlari ham bayon etilgan. Maqolada Alısher Navoiyning «Tarixi Muluki Ajam» asarida qadimgi Eronning Zahhok binni Madoris, Doro binni Dorob, Hurmuz binni Anushirvon kabi zolim shohlar tasvirining tahlili asosida shoirning odil shohlar xususidagi orzulari hamda zamondosh podsho va shahzodalarga o'gitlari tadqiq etilgan. Sharq adabiyotida qadimdan mavjud bo'lgan odil va zolim shohlar haqidagi g'oyalarning rivojida ushbu asarning roli ko'rsatib o'tilgan
\end{abstract} Davlat.

Kalit So'zlar: Alisher Navoiy, Tarix, Ajam, Zolim Shoh, Adolatli Shoh, Shahzoda,

\section{ALİ ȘIR NEVAYİ'NİN “TARİH-İ MÜLUK-İ ACEM” ESERİNDE ZALİM SAHLARIN GÖRÜNTÜSÜ}

Özet

Bilindiği gibi "Tarihi Mulüki ve Acem" Ali Şir Nevai'nin tarihsel eseri ise de bu eserde ulu düşünürün adaletli padişah ve devlet hakkındaki arzuları, zalim padişahlar ve onların zevali hususundaki görüşleri yer almaktadır. Bu makalede Ali Şir Nevai'nin antik İrandaki Zahhok binni Madoris, Dara binni Dārāb, Hurmuzd binni Anuşirvan gibi zalim şahların mezalimi ve zevali üzerindeki yorumları, ayrıca bu hikâyeler üzerinden düşünürün dönemin sultan ve şehzadelerine yollanan öğütleri analiz edilmektedir. Ali Şir Nevai'nin bu çalıșmasının geleneksel Doğu edebiyatının adil padişahlar hakkındaki gayelerinin gelişimi üzerindeki rolünü değerlendirmek için bir girişimde bulunulmuştur.

Anahtar Sözcükler: Ali Şir Nevai, Tarih, Antik İran, Zalim Şah, Adaletli Şah, Şahzadeler, Devlet.

\section{IMAGES OF TYRANT KINGS IN THE WORK OF ALISHER NAVOI "TARIH- I MULUK-I AJAM"}

\begin{abstract}
"Tarih-i Muluk-i Ajam" is a historical work of Navoi, but it also describes views of the great thinker on equitable king and state and about cruel kings. This article analyzes the views of Alisher Navoi on the tyrant kings of ancient Iran, like Zahok binni Madoris, Dara Binni Darab, Khurmuzd Binni Anushirvan and his dreams about equitable kings, as well as advice of the thinker to the kings and princes-contemporaries. Also studied the role of this creativity of Navoi in development of ideas about equitable and tyrannical kings existed in the Oriental literature.
\end{abstract}

Key Words: Alisher Navoi, History, Ancient Iran, Tyrant King, Equitable King, Princes, State.

\footnotetext{
* Semerkant Devlet Üniversitesi Özbek Mümtaz Edebiyatı Bölümü, Semerkant / ÖZBEKİSTAN, el-mek: irodapardayeva1975@gmail.com

(D) ORCID ID: https://orcid.org/ 0000-0002-9248-6188
} 
Adolatli podsholar, ularning amalga oshirgan ezgu ishlari va ularning tarixda qoldirgan nomlari Sharq xalqlari ijtimoiy onggida va, xususan, adabiyotida qanday katta iz qoldirgan bo'lsa, adolatsiz va zolim shohlarning xalqqa qilgan zulmlari ham tarixga ana shunday raqam qilingan. Sharq adabiyotidagi an'analariga ko'ra "adolatli shoh" va "zolim shoh" dilemmasi, antagonizmi ko'pincha qadimgi Eron shohlari misolida ko'rib chiqilgan' ${ }^{1}$.

Butun umrini el yurt farovonligi yo'lida ezgu ishlarga baxsh etgan, ulug' shoir, mutafakkur hamda yirik davlat arbobi Alisher Navoiyning odil hukomronlik to'g'risidagi qarashlari hayotiyligi bilan hamon qadrlidir. Navoiy "Hayrat-ul abror" va "Saddi Iskandariy" dostonlarida o'z orzusidagi odil podshoh timsolini yaratadi va shunday podshohning siyosati tufayli vujudga kelgan kuchli davlatchilik g'oyalarini tarannum etadi. Shu bilan bir qatorda shoir Qur'on g'oyalari asoslangan holda " Podshoh Allohning yordamchisi, Alloh unga bu vazifani to'g'rilik hamollik odillik bajarishni topshirgan" (Alisher Navoiy, 2000: 232), deya ta'kidlaydi.

Navoiy nazaridagi hukmdor mamlakatni adolat bilan boshqarishi, qa'tiyatli bo'lishi, siyosat yuritish va hukm chiqarishda ehtiroslarga berilmay, aql ovoziga quloq tutishi, zarur o'rinlarda kechirishni bilishi, ya'ni nohaqdan tumat bilan aybdor deb topilgan kishilarga adolatpeshalik qilishi, hech qachon qalbida kir, adovat saqlamasligi, xolis bo'lishini, har biri ishini kengash asosida qilishi, doimo el-yurt ahvolidan, kayfiyatdan boxabar bo'lishi, davlat ahamiyatiga molik har bir ishini o'z vaqtida hal qilishini lozim deb hisoblaydi.

Alisher Navoiy davlat va shohlarni ikki qarama-qarshi qutbga - adolatli va adolatsiz tabaqalarga ajratadi. Adolatsiz davlat, shoh insonlarga zulmu zo'rlik olib kelsa, adolatli davlat, shoh kishilarga baxt-saodat keltiradi. Navoiy davlat apparatining yagona mexanizmdan iborat bo'lishini ochib beradi. Shoirning aytishicha, davlat boshlig'i - shoh qanday bo'lsa, uning qo'l ostidagi mulozimlari ham xuddi shunday bo'ladilar. Agar shoh adolatsiz bo'lsa, uning vaziri, beklari, mirshabu qozilari ham adolatsiz bo'ladilar va aksincha, agarda shoh adolatli bo'lsa, uning mulozimlari ham shunga monand bo'lishadi.

Navoiy adolatli shohlarni insonlarga tenglik, ozodlik, do'stlik olib keluvchi birdan-bir kuch deb qarab, shunday hukmdorlarni orzu qiladi, olqishlaydi. Adolatli shoh uning idealiga aylanadi.Alisher Navoiy «Tarixi muluki ajam» asarida adolatli podshohlar haqida so'z yuritar ekan, zolim shohlar tarixiga, o'tmishiga murojaat qilib, ular haqida alohida to'xtalib o'tishni ham lozim ko'radi. U zolim shohlar qismatini tasvirlash, xatolarini qayd etish orqali zamona hukmdorlarini ogohlikka, hushyorlikka chorlaydi. Podshohlarning g'ururga, kayf-safoga, xotirjamlikka, shaxsiy manfaatlarga berilishi oqibatida mamlakat va xalqni yomon ahvolga solib qo'yishini ko'rsatib beradi. Masalan, Bahrom Go'r odil shoh bo'lsa ham sharob ichish va kiyik ovlashni yaxshi ko'rib, shunga berilishi natijasida mamlakatni dushman bosib oladi. Navoiy Bahrom Go'r to'g'risida so'zlab kelib, birdan Husayn Boyqaroning Yodgor Muhammad bilan urush qilib, Navoiy yordamida Hirot taxtini qanday qilib qo'lga kiritganiga o'tadi: “...Abulg'ozi Sulton Husayn Bahodirxon xalladallohi mulkahug'a muyassar bo'ldikim, bu nav ish qildi. Va aning mujmali budurkim, ul hazrat Xuroson taxtin olg'ondakim, hanuz mulk to'xtamaydur erdi, Yodgor Muhammad Mirzokim, mulk vorisi erdi..." (Alisher Navoiy, 2000: 236).

Bu bilan Navoiy tarixiy shoh Bahromni Husayn Boyqaro bilan bog'lab ko'rsatadi. "Sab'ai sayyor" dostonining oxirida Bahrom Go'rni ochiqdan-ochiq Sulton Husayn Boyqaroga o'xshatishini yodga olsak, "Tarixi muluki Ajam"da faqat Bahrom Go'r to'g'risidagina emas, balki hamma podshohlar haqidagi fikrlar, avvalo Sulton, qolaversa, uning o'g'illariga qaratilganligiga ishonch hosil qilamiz.

\footnotetext{
${ }^{1}$ bk. V. Zohidov. Ulug' shoir ijodining qalbi. -T.: O‘zbekiston, 1970; Sarkhosh Curtis, Vesta; Stewart, Sarah. Birth of the Persian Empire: The Idea of Iran, London: I. B. Tauris, 2005. -p. 108; Subtelny, Maria Eva C. E. Bosworth; E. Van Donzel; W. P. Heinrichs; Ch. Pellat, eds. Mīr 'Alī Shīr Nawā'ī. The Encyclopedia of Islam. VII. Leiden-New York, 1993; Bertels E.E. Navoiy. Opit tvorcheskoy biografii. -M-L.: 1948.
} 
Navoiy asarda Zahhok binni Madoris, Doro binni Dorob, Hurmuz binni Anushirvon kabi zolim shohlarning ibratli qismatiga ham alohida to'xtalib o'tadi.

Zahhok binni Madoris haqida dastlabki ma'lumot Firdavsiyning "Shohnoma» asarida uchraydi. Asarda aytilishicha, Arabistonda Mardos degan donishmand podshohning Zahhok degan johil va zolim o'g'li bo'ladi. Zahhok Ahrimanning fitnasi bilan keksa otasini o'ldirib, taxtga chiqadi va Eronni zabt etadi, el-yurtga qattiq zulm qila boshlaydi, qochib ketgan Jamshidni qo'lga tushirib, qatl qiladi. Ahriman kishilarning boshiga solgan zulm va baxtsizliklar bilan qanoatlanib qolmaydi. U iblis Zahhokning oshpazi qiyofasida bo'lib, uning ishonche'tiborini qozonadi. Kunlarning birida iblis Zahhokning ikki yelkasidan bo'sa olishga ruxsat so'raydi. Iblisning sehrli nafasi bilan Zahhokning ikki elkasidan ikkita katta ilon o'sib chiqadi. Iblis tabib qiyofasida Zahhokning dargohiga keladi. U Zahhokka jonini saqlab qolish uchun mamlakatdagi yigitlarni birin-ketin o'ldirib, ilonlarni ularning miyasi bilan boqishini maslahat beradi. Juda ko'plab yigitlar qirilib ketadi. Kova degan temirchining o'n sakkiz o'g'li bo'ladi. Zahhok uning o'n etti o'g'lini o'ldiradi. Navbat o'n sakkizinchi o'g'liga keladi. G'azablangan Kova saroyga boradi. Lekin Zahhokdan ham, uning ayyonlaridan ham najot topolmaydi. U charm peshpandini bayroq qilib, xalqni kurashga chaqiradi. Zahhokga qarshi butun halq kurashga otlanadi, ularga Faridun boshchilik qiladi. Zahhok mag'lub bo'ladi, uning jasadi Damovand tog'ining g' origa mixlab tashlanadi. Faridun taxtga chiqadi (Homidov, 1991: 12-13).

Navoiy bu mifik zolim shoh haqida: “...Zahhok saltanati va zulmi uzoqg'a tortti, andoqkim el aning zulmidin ojiz bo'ldilarkim, aning ikki egnidin andoq maraz paydo bo'lib erdikim, og'rig'ig'a odamzod mag'zidin o'zga hech nima taskin bermas erdi. Ba'zi muarrixlar aning iki egnidin yilonlar chiqib, g'izolari kishi mag'zi erdi, ham debdurlar. Har taqdir bila ul har kun bu jihatdin iki kishi o'lturur erdi... Sipohonda Kova ohangarning bir o'g'lig'a dag'i bor edi, bu jihatdin qatl bo'lib erdi, yana bir o'g'lig'a chek tushti. Ul betahammul bo'lib, qichqirib, elga ko'p ta'nlar qilib Zahhokni so'kti. El dag'i anga muttafiq bo'lub, xuruj qildilar. Dag'i Sipohon volisini o'lturub, Zahhok ustig'a yurdilar. ...Kova ohangar sipohsolor bo'lub, temirchilar beliga bog'lar saxtiyonni yig'och boshig'a bog'lab alam qilib yurib, Zahhokdin chun el ayurulub erdilar, turolmay qochti. Ammo uni qovub iliklab, Faridun ani jazosig'a yetkurdi..." (Alisher Navoiy, 2000: 236) deydi. Navoiy Zahhokni taxtdan ag'darishda yo'lboshchilik qilgan temirchi, mehnatkash xalq vakili Kovaga nisbatan xayrixohlik bildiradi. E'tibor beradigan bo'lsak, muallif Zahhok binni Madoris haqida so'zlar ekan, uning xalq hayoti uchun biror ish qilganligi yoki yangilik yaratganligini qayd etmaydi. U faqat xalqqa zulm-zahmat etkazgan zolim shoh sifatida tasvirlangan, xolos. Navoiy "Saddi Iskandariy" dostonida zolim shohlar haqida shunday deydi:

\section{Yana buki elga solib iztirob,}

Qilur zulmi obodlarni xarob.

Yana ulki, chun zulme mavjud o'lur,

Bori xalq ko'ngliga mardud o'lur.

Ne mardudluqkim, bo'lur el ishi -

Tunu-kun aning jonining qarg'ishi (Alisher Navoiy, 1991: 107).

Navoiy o'z fikrida davom etib shunday yozadi:

Necha zulmkim elga zolim qilur,

O'z-o'ziga ko'prak qilur - haq bilur (Alisher Navoiy, 1991: 108).

Zolim elga qanchalik zulm etkazsa, bilginki, bu zulmlar uning o'ziga undan ham ko'proq bo'lib qaytadi - bu xalqqa ham ayon bo'lgan haqiqatdir. Buning tarixdagi isbotini yuqorida ko'rib o'tilgan zolim Zahhok ibn Madoris qismati misolida kuzatish mumkin. Xalqqa qilingan zulm o'ziga undan ko'proq tarzda qaytib keldi. 
Navoiy "Tarixi muluki ajam"da zolim shohlar qatoriga kayoniylar sulolasidan bo'lgan Doro binni Dorobni ham kiritadi va u haqida shunday yozadi: “...Chun zolim ta'b kishi erdi, zulmidin ulug' el ozurda bo'ldilar". Uning ikki noyibi tomonidan o'ldirilishi sababini ham Navoiy aynan uning zulmida deb biladi: "Doroning iki noibikim, aning zulmidin toriqib erdilar, ani halok qildilar" (Alisher Navoiy, 2000: 215).

Muarrix bu podshoh haqida o'zining “Xamsa"sida alohida to'xtalgan va u to'g'risida kengroq ma'lumot bergan edi. Shu bilan birga, bu tarixiy asarida ham uning tarixini "Saddi Iskandariy"dagi tasviriga nisbatan qisqaroq tarzda bo'lsa-da, yoritib beradi. Iskandar bilan bog'liqlik va ular o'rtasidagi munosabatlarni lo'nda qilib ifodalaydi. Masalan, Doroning o'limi oldidan Iskandarga qilgan uch vasiyati “Saddi Iskandariy"da qo'yidagicha ifodalangan:

Kim: "Ul uch so'zum - uch vasiyatdurur,

Ki izhori ko'nglumga niyatdurur.

Birinchi bukim, ul iki kiynaxoh,

Ki qatlimg'a qo'l sundilar begunoh,

Alar qatlin oyini dod etgasen,

Bu ish birla ruhimni shod etgasen.

Yo'q, andin manga garchi daf'i gazand,

Va lekin sanga bordururur sudmand.

Yana, qavmu xaylimg'a qilma inod,

Ki derlar alarni Kayoniynajod.

Yana Ravshanakkim, qizimdur mening,

Bu kundin nari mensizimdur mening.

Bisotingni ul sham' birla yorut,

Aning shug'lidin xotiringni ovut.

Kivur mehr birla shabistoninga,

Qilib aqd, o'lturt ani yoninga (Alisher Navoiy, 1991: 170-171).

Buni Navoiy "Tarixi muluki Ajam"da shunday ixcham tarzda bayon etadi: "Va Doro Iskandarg'a uch vasiyat qildi. Bir ulkim, Ravshanaknikim, aning qizi erdi, qilg'ay. Bir ulkim, aning qotillarin o'lturgay. Bir ulkim, aning atboikim, Furs muluki naslidin erdilar, qatl qilmag'ay, dag'i rioyat qilg'ay".

Ko'rib o'tganimizdek, Doro obrazi har ikki asarda ham bir xil ko'rinish, bir qismat egasi sifatida namoyon bo'lgan. Navoiy "Xamsa"da u haqida badiiy tafsilotga keng o'rin bergan bo'lsa, "Tarixi muluki Ajam”da esa uning qisqacha bayoni bilan cheklanadi. 
Navoiy asarda yuqorida ko'rib o'tilgan zolim shohlardan tashqari Bahrom binni Shopur ismli zolim shoh haqida ham alohida to'xtalib o'tadi: "Banokatiy debdurkim, aning zulmidin anga el g'avg'o qildilar va ul g'avg'oda halok bo'ldi" (Alisher Navoiy, 2000: 233). Bunda Navoiy xalq kuchi va ahamiyatini ko'rsatib beradi.

Umuman, Alisher Navoiy "Tarixi muluki Ajam" asarida odil va zolim podshohlar haqida so'z yuritar ekan, ulardan zamona shohlari ibrat, o'rnak olishi lozimligini nazarda tutadi. Odil shohlarning xalqqa qanchalik foydasi tegishi, yurtning obod bo'lishi ularning odilona va oqilona faoliyatiga bog'liq ekanligi, qilingan zulm eng avvalo, shohning o'ziga qilingan zalolat ekanligini ko'rsatib beradi. Navoiy bu orqali nafaqat mamlakat egalarini, balki oddiy insonlarni ham ogohlikka chaqiradi. Ikkinchidan, asarni o'qib chiqqan kitobxon ilmiy-tarixiy jihatdan ma'lum bilimga ega bo'ladi, Eron shohlari tarixidan xabardor bo'ladi. Shuningdek, tarbiyaviy jihatdan, ibrat nuqtai nazaridan ham ma'lum xulosa va yakuniy mulohazalar hosil qiladi .

\section{Adabiyotlar}

Alisher Navoiy (1991). Saddi Iskandariy. Toshkent: G'.G'ulom nomidagi NashriyotMatbaa Birlashmasi. Fan.

Alisher Navoiy (2000). Mukammal asarlar to'plami. Yigirma jildlik. 16-jild, Toshkent:

BERTELS, E. E., (1948). Navoiy. Opit Tvorcheskoy Biografii. M-L.

HOMIDOV, H., (1991). Shohnoma"ning Shuhrati. Toshkent: O'zbekiston.

ISHOQOV, Y., (1967). Navoiy va Xusrav, O'zbek Tili va Adabiyoti. 4-son, B. 15.

SARKHOSH CURTIS, V., STEWART, S., (2005). Birth of the Persian Empire: The Idea of Iran. London: I. B. Tauris.

SUBTELNY, M. E., BOSWORTH, C. E., (Eds. E. Van DONZEL, W. P. HEINRICHS, Ch. PELLAT) (1993). Mìr 'Alī Shìr Nawā't̄. The Encyclopedia of Islam. VII. Leiden-New York.

ZOHIDOV, V., (1970). Ulug ‘ Shoir Ijodining Qalbi. T.: O‘zbekiston. 